\title{
DEVELOPMENT OF AN ADVANCED METHOD OF VIDEO INFORMATION RESOURCE COMPRESSION IN NAVIGATION AND TRAFFIC CONTROL SYSTEMS
}

\author{
Serhii Yevseiev \\ Department of Cybersecurity and Information Technology \\ Simon Kuznets Kharkiv National University of Economics \\ 9-A Nauky ave., Kharkiv, Ukraine, 61166 \\ serhii.yevseiev@hneu.net \\ Ahmed Abdalla \\ Department of Flight Operations \\ Flight Academy of the National Aviation University \\ 1 Dobrovolskoho str., Kropyvnytskyi, Ukraine, 25005 \\ Ahmedalassam90@gmail.com \\ Serhii Osiievskyi \\ Department of Mathematical and Software Control Systems ${ }^{1}$ \\ stiv161272@gmail.com \\ Volodymyr Larin \\ Department of Mathematical and Software Control Systems ${ }^{1}$ \\ l_vv83@ukr.net \\ Mykhailo Lytvynenko \\ Department of Mathematical and Software Control Systems ${ }^{1}$ \\ avtris@ukr.net \\ ${ }^{1}$ Ivan Kozhedub Kharkiv National Air Force University \\ 77/79 Sumska str., Kharkiv, Ukraine, 61023
}

\begin{abstract}
The Earth's aerospace monitoring (ASM) systems use state-of-the-art integrated information technologies that include radio-based detection and surveillance systems using telecommunications. One of the main tasks of ASM systems is to increase the efficiency of decision-making necessary for the timely prevention, detection, localization and elimination of crisis situations and their probable consequences. Modern conditions impose stricter requirements for efficiency, reliability and quality of the provided video data. To ensure compliance with the requirements, it is necessary to provide the appropriate capabilities of the onboard equipment. On the basis of the existing information and communication systems it is necessary to carry out: continuous or periodic assessment of a condition of objects of supervision and control; continuous (operational) collection, reception, transmission, processing, analysis and display of information resources. It is proposed to use UAVs (unmanned aerial vehicles) as a means to perform ASM tasks. The time of organizing communication sessions and delivery of information should vary from a few seconds to 2.5 hours. Untimely processing and delivery of a specific information resource in the management process leads to its obsolescence or loss of relevance, which contributes to erroneous decisions.

One way to reduce time is to encode the data. To do this, it is proposed to use video compression algorithms. However, based on the analysis of the possibility of modern methods of video information compression, taking into account the specifics of the onboard equipment of the UAV, the coding problem is not completely solved.

The research results show the expediency of using an improved method of video information compression to reduce the computing resources of the software and hardware complex of the onboard UAV equipment and to ensure the requirements for efficiency and reliability of data in modern threats to ASM systems as a whole.
\end{abstract}

Keywords: aerospace monitoring, informatization, onboard system, redundancy, video information resource. 


\section{Introduction}

In modern on-board systems in the process of shooting and the formation of information resources are widely used cameras based on solid-state matrices, the so-called "charger connection devices" (CCD). The parameters of the CCD matrices are determined by the class and level of the tasks to be solved. The main technical characteristic of digital optical systems on board equipment is their resolution, which depends on the dimension of the matrix device with a charger, on which after the lens is projected the resulting static element of the video information resource.

The on-board equipment of the UAV has its own features, such as: limited size, patent restrictions on algorithms and programs, insufficient capacity to process information resources in the process of working in a time mode close to real.

Currently, the onboard equipment of the UAV does not provide the ability to set the omnidirectional signal. Directional terrestrial antennas are required to accompany the device along the line of sight. But for urban conditions, conditions of poor visibility, mountainous terrain, there are restrictions on direct visibility. The use of other approaches, namely satellite groups and cellular communication systems, is too expensive.

Modern on-board equipment of the UAV, in solving the problems of ASM has shortcomings that directly affect the efficiency of decision-making in the process of navigation and traffic control:

1) On-board equipment of the system of collection, processing and transmission of information on existing and developed systems of navigation and traffic control does not provide complete recording and transmission of all video information received from one line of photocells in one second:

- for UAV systems of Ukraine, as an example "MS-2-8" with a resolution of $7.8 \mathrm{~m}$ per $1 \mathrm{sec}-$ ond on communication channels on the ground segment can be transmitted no more than $30 \%$ of all taken for 1 second information;

- for systems of the "Sich-3-O" type with high resolution $(0.9 \mathrm{~m})$ for $1 \mathrm{~s}$ on communication channels no more than $15 \%$ of information can be transferred accordingly;

- with increase in resolution of the onboard equipment to $0,5 \mathrm{~m}$ transfer on communication channels on a terrestrial segment no more than $3 \%$ of all removed information is possible; to $18 \mathrm{~min}$.

- the minimum transmission time of the required amount of information varies from $3 \mathrm{~s}$

2) The minimum transmission time of one frame of digital video from the UAV acquires the following values:

- for a frame size of $5120 \times 5120$ elements - up to 12 minutes;

- for a frame size of $9420 \times 9420$ elements - up to 2.5 hours.

Thus, the existing capabilities of the on-board equipment for data processing and transmission, in solving the problems of ASM, do not provide the necessary efficiency for digital image processing.

Improper delivery of video information resources in the process of air traffic control leads to its aging and loss of relevance and contribute to erroneous and untimely decisions. This affects the effectiveness of operations to eliminate, localize and prevent emergencies.

Therefore, there is a need to reduce the processing time and transmission of video data in telecommunications aerospace monitoring systems. One of the effective ways to increase the efficiency of information delivery is to reduce the amount of processed and transmitted data. Reducing the amount of information while maintaining a given degree of reliability minimizes the time of information transmission through communication channels and reduces the intensity of information exchange, reduces the time of formation of its graphical model in display devices, and increases productivity and quality of the entire telecommunications system.

Thus, to implement processes to improve processing efficiency, provide a given quality and completeness of video information resources, it is necessary to reduce redundancy in the process of working with dynamic images, taking into account the design features of onboard UAV equipment to reduce decision-making time. 


\section{Literature review and problem statement}

In works [1-3] in the study of the processes of management of high-risk objects, as well as the localization and elimination of emergencies in recent years, an assessment of the requirements for resolution. This shows that the situation is changing dynamically, it is difficult to choose the objects of observation before the departure of the aircraft. But at the same time, the limitations of UAV communication systems, which directly affect the time of decision-making, are not taken into account.

The authors in [4-6] show that the time of delivery of video information from the UAV is influenced by the following factors (limitations) caused by the peculiarities of this method of obtaining information, but it was not taken into account that:

1) limited mass and size characteristics of the UAV. More than $70 \%$ of the total initial mass of the UAV is allocated to the engine;

2) limited power capabilities of power supplies. It should be borne in mind that the engine requires about $90 \%$ of total power, and to provide avionics and communication systems is allocated $10 \%$;

3) conducting video photography at a certain altitude and flight speed of the UAV. This imposes an additional time limit $t_{l}^{a)}$ between the formation of adjacent images, which is directly proportional to the height and speed of the UAV, on which the information is taken;

4) requirements for weather conditions, namely the limited flight time caused by high precipitation density and temperature regime (performance of the tasks should be carried out at a temperature of $-40{ }^{\circ} \mathrm{C}$ to $+50{ }^{\circ} \mathrm{C}$ ).

Thus, it is possible to conclude that currently, in terms of increasing requirements for efficiency, quality and completeness of video information, the existing methods of processing onboard UAV equipment do not allow to perform the tasks in full, so it is proposed to use an improved method of video information compression in systems navigation and traffic control, which reduces the time of information transfer to the desired level with a given quality.

\section{The aim and objectives of research}

The aim of the article is to develop an improved method of compression of video information resources in navigation and traffic control systems, taking into account the limitations of data processing on UAV board.

To achieve the stated aim it is necessary to solve the following objectives:

- to analyze compression methods and requirements of video information resource processing equipment on board aerospace monitoring facilities;

- to develop an improved method of video information compression in navigation and traffic control systems.

\section{Analysis of compression methods and requirements for equipment for processing video information resources on board of aerospace monitoring facilities}

To ensure the timely delivery of video information resources, it is necessary to take into account the high-speed capabilities of communication channels. To do this, data compression algorithms are used.

Approaches to creating methods of compact representation can be divided into three classes depending on the requirements for the quality of reconstructed images, which are presented in Fig. 1.

Methods with irreversible changes form the first class, as shown in [7-9]. In the process of compression technologies of morphological processing of video information are used. On the contrary, the second class consists of methods that perform processing without error, as shown in $[10-12]$.

The rms value of the distortion level is close to zero, $\sigma=0$, and the peak signal-to-noise ratio, respectively $Q_{k} \rightarrow \infty$. Representatives of this class of methods are methods of series length coding (SLC), dictionary methods LZW, Huffman coding, arithmetic coding (AC), polyadic coding method (PCM). The intermediate place between the two previous classes of methods is 
occupied by the methods of the third class. The basic component of the methods of this class is pre-treatment based on the use of orthogonal transformations, wavelet systems and affine transformations. Technologies based on such approaches are implemented in JPEG (discrete cosine transform (DCT)) and JPEG2000 (wavelet transform) formats. These formats allow to present the image in a processed form both without loss of quality (loss-less mode) and with loss of quality.

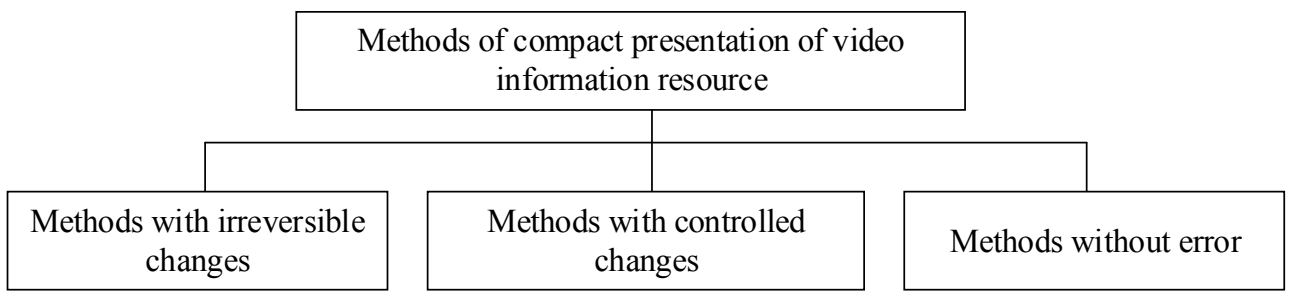

Fig. 1. Methods of compact presentation of video information resource

In works [13-15] at an estimation of methods of compact representation of video of an information resource the following lacks of the onboard equipment of the UAV were revealed:

- insufficient size of antennas due to the small size of the device;

- limited capabilities of data transmission equipment, which is due to insufficient energy resources of the onboard complex.

The consequence of such shortcomings is the problems associated with the organization of data transmission, namely the lack of energy capacity to maintain the necessary speed for the transmission of video information resources.

Second and third class methods are used to organize data processing in aerospace monitoring systems. Image types are determined depending on the average length of a series of identical elements and the correlation coefficient.

Analysis of the characteristics of compression processes based on error-free methods allows to conclude that:

- time for compression, recovery, and image transfers is about several minutes;

- time of transmission of small sizes images $(800 \times 600$ elements) in volume of $11 \mathrm{Mbit}$ by means of mobile radio communication $\left(R_{n}=9,6 \mathrm{Kbps}\right)$ will reach several tens of minutes.

These shortcomings are explained both by low values of the degree of compression of saturated images (on average up to 2 times), and additional time delays for processing. Here the greatest complexity of processing processes corresponds to the methods of arithmetic coding and dictionary methods LZW.

Compression technologies with controlled quality loss allow to further increase the degree of compression of aerospace monitoring images in comparison with error-free methods, which is shown in $[14,16]$, taking into account the possibility of reducing psychovisual redundancy.

Studies allow to conclude that for methods with controlled loss of quality there are certain features, namely:

- compression degree of the video information resource of aerospace monitoring is on average 3.5 times higher than the degree of compression of methods without error. However, such a gain is achieved by making errors in the video image being processed. Further reduction of the accuracy of digitization of the transformant component leads to a deterioration in the quality of the reconstructed images to the level of $25-35 \mathrm{~dB}$;

- when increasing the size of the images to $4096 \times 3072$ elements, the total time for their processing by onboard radio equipment and transmission over the communication channel increases to a few minutes, as shown in [16].

Such results limit the application of the considered technologies on the onboard equipment of the UAV. The identified shortcomings for the methods of the third class are due to the following reasons: 
1) limited compression ratio for JPEG and JPEG 2000 methods for mode $Q_{k} \geq 40 \mathrm{~dB}$. The greatest reduction in the degree of compression (up to 2.5 times) is manifested in the processing of saturated images of aerospace monitoring;

2) implementation of two-dimensional DCT and DWT-transformations for onboard computer systems is associated with the need to spend from 10 to $70 \%$ of the total processing time, as shown in [19]. The time for their execution in the process of converting video images can reach tens of seconds. This is due to the large number of operations;

3) the basic methods for eliminating statistical redundancy in transformed images are Huffman codes and arithmetic coding, for which there are certain properties, namely:

- in the case of processing saturated video images of aerospace monitoring in the mode of controlled quality, short zero chains are generated. Hence, the lengths of such chains will have a uniform distribution and, as a consequence, a small amount of statistical redundancy. This leads to a sharp decrease in the compression ratio of video data;

- significant computational costs, reaching $90 \%$ of the total amount of computations allocated to the process of video data compression. This is due to both the processing of individual binary structures of the transformant components, and the performance of several multiplication operations to process a single element. The number of operations to perform arithmetic coding in the space of bit planes, formed on the basis of DCT and DWT components, may exceed the number of operations to perform transformations (for modes that provide high image quality);

- there are no mechanisms for implementing parallel processing schemes;

- there is a need to use marker code sequences that separate uneven code combinations of statistical codes. This increases the size of the compressed view.

4) low resistance of efficiency of series lengths formation processes to increase of saturation degree and to errors in the communication channel.

These shortcomings in the operation of telecommunications aerospace monitoring system are associated with the following difficulties:

- increasing the amount of processed and transmitted video data (up to $10^{5} \mathrm{Gbps}$ );

- limited tactical and technical capabilities of information and communication technologies in existing and promising aerospace systems.

Required time and set level $d_{i}$ decryption of information about a typical monitoring object (resolution) depends on the level of complexity. Thus, the information required by the lower level operator is characterized by high detail of each object, while for higher level operators such detail is not important. Characteristic detail $d_{i}$ on the ground (resolution), which provides shooting equipment on board the UAV, depending on the height of the shooting, focal length, the number of elements in the matrix, affects the probability $P_{o d}$ observation object detection. Magnitude $P_{o d}$ is estimated as:

$$
p_{o d}=\exp \left[\frac{\ln \alpha}{\lg \frac{l+k}{l-k}}\left(\frac{d}{d_{\delta}}\right)^{2}\right]
$$

where $d_{p}$ - the required resolution (characteristic detail in the field, taking into account the level of decryption of images of a typical object of observation), which is determined on the basis of the approach using the specified tabular values; $d$ - real resolution for onboard camera equipment, taking into account the height of shooting $H$, focal length $f$, and recognizability $R$ of iconic system, that is: $d=H / 2 R f$. Dependence of the value of resolution $d$ from the height of shooting $H$ and frame size $n_{p} \times n_{c}$ (CCD size) at a given focal length.

Factors that lead to limiting the effectiveness of UAV communication systems are shown in Fig. 2. This leads to limited time $t_{c s}^{a}$ communication session between the UAV and the recipient of information. 


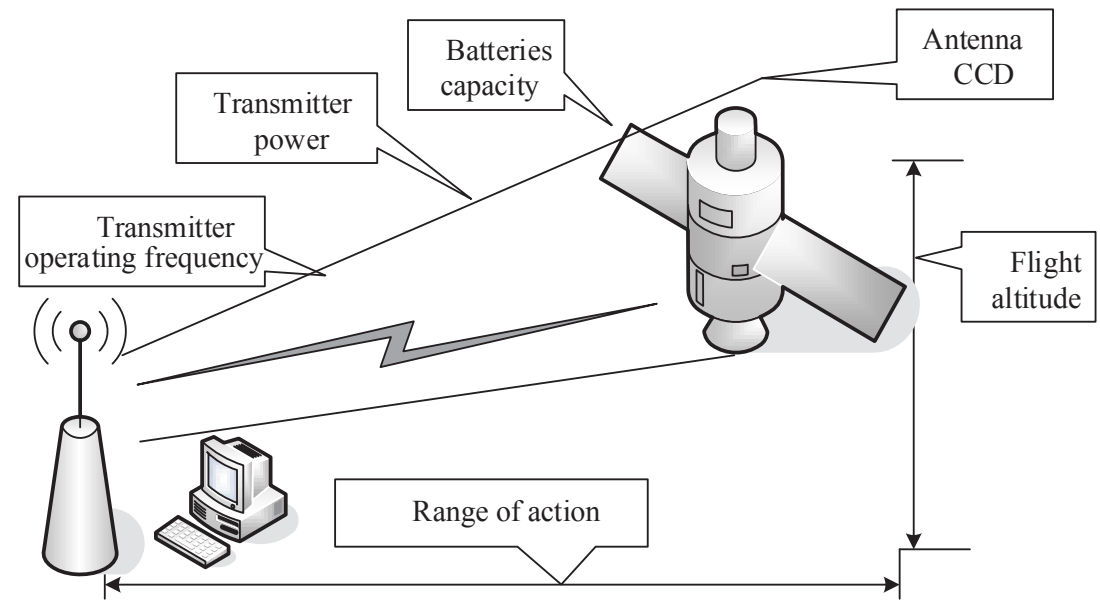

Fig. 2. Factors affecting the efficiency of the UAV communication system

Limited capabilities for the performance of onboard data processing equipment lead to limitations in conducting complex computational processes on board the UAV.

The process of organizing aerospace monitoring using navigation and motion control systems and UAVs is characterized by the following indicators:

a) resolution is required to solve the corresponding class of problems, namely the level of decoding the video image of a typical object of observation (detection, recognition by type, recognition by class, recognition by type and analysis of the object of observation), i. e.:

$$
T(V)_{d}>T(V)_{d}^{(\mathrm{g})}
$$

where $T(V)_{d}^{(\mathrm{g})}$ - the required time to prove the video data of a given volume equal to $V$ bits (determined by the peculiarities of the control process);

b) allowable time $t_{l}^{a}$, is set taking into account the limited time between the formation of adjacent images, i. e.:

$$
T(V)_{d}>t_{l}^{(a)}
$$

Thus, it is necessary to ensure high resolution, the presence of several video cameras, the need for timely proof of reliable information, up to a time close to real. This approach allows to increase the efficiency of crisis management. At the same time, the UAV-based navigation and traffic control system must ensure compliance with strict requirements and reliability during the processing and transmission of video information resources.

Digital volumes of $V$ images generated by cameras on board the UAV are estimated by the following formula:

$$
V=\sum_{i=1}^{v_{c p}}\left(n_{p} \times n_{c} \times \log _{2} Z\right)
$$

where $V_{c p}$ - the number of color planes involved in the formation of a full-color image; $Z$ - the number of bits in the formation of the matrix element; $n_{p}, n_{c}$ - respectively, the number of rows and columns in the image frame.

Thus, expression (3) allows to estimate the digital volumes $V$ of video images generated by the camera on board the UAV, which can reach a volume of the order of hundreds of Mbit and even Gbit.

For known speed values $R_{n}$ data transfer, time $T(V)_{d}$ data delivery, a volume equal to $V$ bits from the UAV is determined by time $T(V)_{p t}$ of preparation and transmission of data over the communication channel and is evaluated by the formula: 


$$
T(V)_{d}=T(V)_{p t}=V / R_{p},
$$

where $R_{n}$ - data rate on the communication channel from the UAV.

Improving the efficiency of proof of video information at a given quality using on-board aerospace monitoring in the process of navigation and traffic control can be described by the following expressions:

$$
\begin{aligned}
T(V)_{d}=T(V)_{p}= & V / R_{p} \leq \min \left\{T(V)_{p}^{(r)} ; t_{m c}^{(a)} ; t_{c s}^{(a)}\right\} ; \\
& \left\{\begin{array}{l}
V \geq V^{(r)} ; \\
d \leq d_{p} ; \quad q \geq q^{(r)}, \\
R_{t} \leq R_{t}^{(r)} ;
\end{array}\right.
\end{aligned}
$$

where $V^{(r)}, R_{t}^{(r)}, q^{(r)}$ - the required values, respectively, for the amount of transmitted video data, transmission speed and quality of the reconstructed video information resource on the receiving side.

When developing an advanced method of compression of video information resources, it is necessary to take into account the presence of restrictions on the processes of their processing on board aerospace monitoring (UAV), namely shown in [20]:

1. The presence of restrictions on the class of the processed information resource, i. e. $0,3 \leq r \leq 0,7, \gamma_{a v}=2$ and $0,9 \leq r, \gamma_{a v}=4$, where $r$ - normalized correlation coefficient, $\gamma_{a v}$ - the average length of a series of identical elements. Aerospace monitoring is characterized by the formation of images saturated with details. This leads to the need to develop methods for changing the initial amount of information based not only on the elimination of probabilistic and statistical redundancy, but also other types of redundancy. In our case, structural.

2. There are restrictions on the speed of data transfer from the UAV on the one hand, and increasing the size of images on the other. This leads to the need to further increase the degree of change in the initial amount of information $A_{m i}$ pictures (according to formula 6):

$$
A_{m i}=V / V_{m i} \geq A^{(r)}
$$

where $V_{m i}-$ the volume of the modified image.

The magnitude of the degree of change of the initial state according to the method of compression on board the UAV must be not less than necessary $H^{(r)}$.

3. Existence of restrictions on energy resource of the onboard equipment. In turn, this imposes additional restrictions on the speed of machine operations to implement the processes of eliminating redundancy and reconstruction. Thus, there are restrictions on the computational complexity of compression algorithms (on the number and type of operations $H_{c m}^{(r)}$ ) and recovery (on the number and type of operations $H v^{(n)}$ ) of video data.

The type of computational operations means arithmetic (addition, multiplication, division) and non-arithmetic (comparison, reading or writing to a storage device) operations, as shown in [21]. In terms of time characteristics, let's obtain the time $T_{k}$ accordingly (according to formula 7 ) for compression and time $T_{v}$ (according to formula 8 ) to restore the video information resource:

$$
\begin{aligned}
& T_{k}=z_{+;-}^{(k)} / R_{+;-}+z_{\times ;+}^{(k)} / R_{\times ;+}, \\
& T_{v}=z_{+;-}^{(v)} / R_{+;--}+z_{\times ; ;}^{(v)} / R_{\times ;-},
\end{aligned}
$$

where $z_{\times ;-}^{(k)}, z_{x ;-}^{(k)}-$ the total number of addition (subtraction) and multiplication (division) operations, respectively, spent on image compression; $z_{\times ;-}^{(v)}, z_{\times ;-}^{(v)}-$ the total number of addition (subtraction) and multiplication (division) operations, respectively, spent on image restoration; 
$R_{+;-}, R_{x_{i}:-}-$ execution speed of operations of addition (subtraction) and multiplication (division) accordingly.

4. Presence of spatial distance of control and notification centers from objects of control. Given the lack of a priori information and the dynamics of change in the situation, there is an additional restriction on the nature and degree of distortion in the process of compression of the video information resource.

The nature of the errors means the properties of controllability or irreversibility of loss of quality of the restored video information resource. The degree of quality change in the reconstructed images is determined on the basis of $Q_{k}$ peak signal-to-noise ratio (PSNR). This indicator takes into account the standard error of the deviation of the elements $e_{i j}^{\prime}$ the restored image relative to the elements $e_{i j}$ original image. Taking into account the restrictions on the degree of loss of quality of the restored images, the condition (according to formula 9) must be met:

$$
q_{c} \geq q^{(r)}
$$

at

$$
q_{c}=20 \lg \left(\frac{255}{\sqrt{\sum_{i=1}^{n_{\delta}} \sum_{j=1}^{n_{\tilde{n}}}\left(\stackrel{\circ}{i j}_{i j}-\dot{a}_{i j}^{\prime}\right)^{2} / n_{r} n_{c}}}\right),
$$

where $n_{r}, n_{c}$ - respectively, the number of rows and columns in the image frame; $q^{(r)}$ - the required value of the peak signal-to-noise ratio.

5. Ability to localize and self-correct distortions caused by errors in code combinations when transmitting them over the communication channel, as shown in [22].

The disadvantages of existing video data compression technologies on board the UAV in the mode of image transmission in the time close to real are as follows:

a) video image shifts due to the influence of a rapid change in the trajectory of the UAV (for example, gusts of wind) on the processing of video information resources, i. e. the speed of a sharp change in the trajectory of the UAV is greater than the speed of information processing;

b) solving operational tasks (search and assessment of the situation) of aerospace monitoring of components of the tactical level (distributed population, distributed vehicles, aircraft at base airfields) is problematic due to insufficient frame size (limited resolution).

Therefore, the additional reduction of the compact presentation of the video information resource on board the UAV will provide additional opportunities to improve the resolution or improve the quality of the restored video images. At the same time it is necessary to consider restrictions on a time resource on data processing.

Thus, as a result, the identified limitations that affect the total time for processing video data using on-board equipment are investigated. Therefore, taking into account these features, an improved method of video information compression in navigation and traffic control systems was proposed.

\section{Development of an improved method of video information compression in navigation and traffic control systems}

The task of improving the method of compression of video information resource in navigation and traffic control systems is based on the fact that the reduction of redundancy occurs at the stage of encoding information by reducing the number of operations for processing and transmission over the communication channel. The improvement of the compression method occurs at the stage of information reconstruction on the receiving side.

Fig. 3 shows a block diagram of the proposed improved method of compression of video information resources in navigation and traffic control systems. 


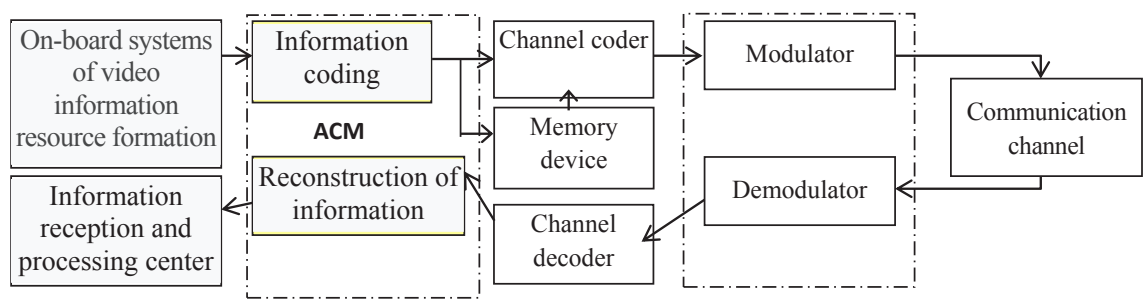

Fig. 3. The structure of the data processing and transmission system using an improved method of presenting a video information resource

The main stages of improving the method of compression of video information resources in navigation and traffic control systems:

1. Providing time $T\left(V_{k}\right)_{p t v}$ processing and transmission of video information from onboard aerospace monitoring so that the condition is met by the formula:

$$
T\left(V_{k}\right)_{p t v}=T_{k}+T\left(V_{k}\right)_{p}+T_{v} \leq \min \left\{T(V)_{p t v}^{(r)} ; T^{(a)}\right\}
$$

subject to the following restrictions, which are defined by the expressions:

$$
\left\{\begin{array}{l}
r<0.5 \& l_{c}=2 ; \\
0.5 \leq r<0.8 \& l_{c}=4 ;
\end{array} ;\left\{\begin{array}{l}
v(V)_{k} \leq v(V)_{k}^{(r)} ; \\
h \geq h_{r},
\end{array} ;\left\{\begin{array}{l}
R_{k} \leq R_{k}^{(r)} ; \\
W \geq W_{r},
\end{array}\right.\right.\right.
$$

where $W$ - the number of binary bits to represent the original digitized image in bits corresponding to the required degree of resolution $d_{0}$ for a specified focal length and video height; $T\left(V_{k}\right)_{p}-$ the transmission time of the processed video data by the communication channel is determined by the formula:

$$
T_{n}=V / A_{m i} R_{n},
$$

where $T(V)_{p t v}^{()}$- the required time to prove the video data of a given volume $V$, taking into account the peculiarities of the process of crisis management; $T^{(a)}$ - the allowable time for delivery of video data from the UAV, taking into account the restrictions on the power of the onboard equipment, the time of the communication session and the time between the formation of neighboring frames is determined by the expression:

$$
T^{(a)}=\min \left\{t_{c s}^{(\partial)} ; t_{m c}^{(\partial)}\right\}
$$

where $t_{c s}^{(a)}$ - the time allowed for the communication session between the UAV and the recipient of the information; $t_{m c}^{(a)}-$ the time between the formation of adjacent personnel on board the UAV, taking into account the speed and altitude of the UAV.

2. Video delivery time should include the additional cost of image compression and recovery processes, as shown in [23].

From this it is possible to conclude that the reduction of redundancy occurs at the stage of encoding information by reducing the number of operations for processing and transmission over the communication channel $T\left(V_{k}\right)_{p t v}$ compactly presented video data, i. e.:

$$
T\left(V_{k}\right)_{p t v} \rightarrow \min
$$

within the constraints given by expressions (11).

To do this, within the limits of (11) must meet one or two conditions (13), (14):

$$
A_{m i} \geq A^{(r)}
$$




$$
A_{m i}+T_{v}<V / R_{p}
$$

Fulfillment of conditions (13) and (14) is a mandatory requirement for the advanced method of compression (AMC) of video information resource in navigation and traffic control systems.

\section{Discussion of the results of research on compression in navigation and traffic control systems}

The required time and the set level of resolution of the onboard equipment of modern UAVs depend on the class of tasks. The delivery time of the information resource with the help of onboard equipment depends on: the method of shooting and formation of information packages on board; required image resolution; the height of shooting objects of interest, focal length and flight speed of the UAV.

Limitations of research are: improvements occur at the stage of coding information by reducing the number of operations for processing and transmission of information over the communication channel, with mandatory control over changes in the quality of video information resources under the conditions specified by formulas (11), (13), (14).

Compression and recovery processes are organized with a controlled loss of quality. Controlled loss of quality means the possibility of methods to ensure the required quality of the reconstructed image, up to providing a mode corresponding to the methods of the second class. An important role in the compression process for such methods is played by the amount of eliminated psychovisual redundancy. The presence of psychovisual redundancy is due to the peculiarities of the perception of images by the human visual system. As a result of the reduction of psychovisual redundancy, as a last resort, critical processes can occur, when in the process of image reconstruction it will be partially or completely destroyed.

Adjustment of quality losses and degree of compression is carried out as a result of:

- zonal and threshold selection and coding of transformant components;

- quantization of the transformant component;

- filtration on the receiving side.

The use of an advanced method of video information compression provides:

- increase free space on memory devices, reduce the cost of their purchase; reducing the likelihood of data loss on the buffer storage device;

- reduction of time of data transmission on communication channels $(\mathrm{CC})$; reduction of requirements to technical parameters of communication channels (data transfer rate); reducing the number of communication channels;

- improving the quality of displayed information; reduction of time of perception of information; reduce video recovery time.

\section{Conclusions}

As a result of research of compression methods and requirements of equipment for processing video information resources on board aerospace monitoring, it was found that in the process of shooting video images are formed, which have a high degree of saturation with small details, color differences and have a significant level of information. It is needed to increase the frame size to provide more detailed video images based on the shooting height. At the same time, the volume of video images generated on the onboard equipment can reach up to hundreds of Mbit. This reduces the efficiency of video information in navigation and traffic control systems.

The proposed improved method of video information compression in navigation and traffic control systems takes into account such limitations as: mass and size characteristics of the UAV, power of power supplies, the time between the formation of neighboring frames. This approach allows to reduce the computing resources of the software and hardware complex of the onboard equipment of the UAV, to reduce the time for the transmission of video data to ground, to ensure the requirements for efficiency and reliability of data in today's threats to ASM systems in general. 


\section{References}

[1] Larin, V. V., Yerema, D. V., Bolotska, Yu. O. (2019). The reasoning of necessity enhancing video privacy in conditions of providing the quality of the video information service provided in virtual infocommunication systems. Science and Technology of the Air Force of Ukraine, 2 (35), 158-162. doi: https://doi.org/10.30748/nitps.2019.35.20

[2] Qassim,H., Verma, A., Feinzimer, D. (2018). Compressed residual-VGG16CNN model for big data places image recognition. 2018 IEEE 8th AnnualComputing and Communication Workshopand Conference(CCWC). doi:https://doi.org/10.1109/ccwc. 2018.8301729

[3] Zakharchenko, M. V., Hadzhyiev, M. M., Basov, V. Ye., Martynova, O. M. et. al.(2009). Systemy peredavannia danykh. Vol. 1: Zavadostiyke koduvannia. Odessa: «Feniks», 406.

[4] Tyurin, V., Martyniuk, O., Mirnenko, V., Open'ko, P., Korenivska, I. (2019). General Approach to Counter Unmanned Aerial Vehicles. 2019 IEEE 5th International Conference Actual Problems of Unmanned Aerial Vehicles Developments (APUAVD). doi: https://doi.org/10.1109/apuavd47061.2019.8943859

[5] Proekt Zakonu pro vnesennia zmin do deiakykh zakonodavchykh aktiv Ukrainy shchodo derzhavnoho rehuliuvannia kosmichnoi diyalnosti No. 1071 vid 29.08.2019. Available at: https://w1.c1.rada.gov.ua/pls/zweb2/webproc4_1?pf3511=66298

[6] VNI Forecast Highlights. Available at: http://www.cisco.com/c/en/us/solutions/service-provider/visual-networking-index-vni/vni-forecast.html

[7] Pavlenko, M., Kolmykov, M., Tymochko, O., Khmelevskiy, S., Larin, V. (2020). Conceptual Basis of Cascading Differential Masking Technology. 2020 IEEE 11th International Conference on Dependable Systems, Services and Technologies (DESSERT). doi: https://doi.org/10.1109/dessert50317.2020.9125024

[8] Li, L. (2015). The UAV intelligent inspection of transmission lines. Proceedings of the 2015 International Conference on Advances in Mechanical Engineering and Industrial Informatics. doi: https://oi.org/10.2991/ameii-15.2015.285

[9] Gonzales, R. C., Woods, R. E. (2002). Digital image processing. New Jersey, 793. Available at: http://web.ipac.caltech. edu/staff/fmasci/home/astro_refs/Digital_Image_Processing_2ndEd.pdf

[10] Kharchenko, V., Mukhina, M. (2014). Correlation-extreme visual navigation of unmanned aircraft systems based on speed-up robust features. Aviation, 18 (2), 80-85. doi: https://doi.org/10.3846/16487788.2014.926645

[11] Telekomunikatsiyni systemy ta merezhi. Vol. 1. Struktura y osnovni funktsiyi. Available at: http://www.znanius.com/ 3534.html

[12] Pavlenko, M. A., Timochko, A. I., Korolyuk, N. A., Gusak, M. Y. (2014). Hybrid model of knowledge for situation recognition in airspace. Automatic Control and Computer Sciences, 48 (5), 257-263. doi: https://doi.org/10.3103/s014641 1614050083

[13] Wang, S., Zhang, X., Liu, X., Zhang, J., Ma, S., Gao, W. (2017). Utility-Driven Adaptive Preprocessing for Screen Content Video Compression. IEEE Transactions on Multimedia, 19 (3), 660-667. doi: https://doi.org/10.1109/tmm.2016.2625276

[14] Tkachov, V. M., Tokariev, V. V., Radchenko, V. O., Lebediev, V. O. (2017). The Problem of Big Data Transmission in the Mobile «Multi-Copter - Sensor Network» System. Control, Navigation and Communication Systems, 2, $154-157$. Available at: http://openarchive.nure.ua/bitstream/document/4536/1/suntz_2017_2_40.pdf

[15] Krasnorutskij, A., Tristan, A., Kharchenko, N. (2014). The Problem Aspect of Control of Bit Speed of the Video Stream in Telecommunication Networks. International Conference TCSET'2014 “Modern problems of radio engineering, telecommunications, and computer science”. Lviv, 533-534. Available at: https://www.researchgate.net/publication/301793981_ Developing_PC_Software_Project_Duration_Model_based_on_Johnson_transformation

[16] Mistry, D., Modi, P., Deokule, K., Patel, A., Patki, H., Abuzaghleh, O. (2016). Network traffic measurement and analysis. 2016 IEEE Long Island Systems, Applications and Technology Conference (LISAT). doi: https://doi.org/10.1109/ lisat.2016.7494141

[17] Savchenko, V., Tolubko, V., Berkman, L., Syrotenko, A., Shchypanskyi, P., Matsko, O. et. al. (2020). Model of an alternative navigation system for high-precision weapons. The Journal of Defense Modeling and Simulation: Applications, Methodology, Technology, 154851292092195. doi: https://doi.org/10.1177/1548512920921955

[18] Saiko, V., Nakonechnyi, V., Toliupa, S., Tiurin, V., Andreeva, K., Maratkyzy, K. (2019). Realization of LEO-systems with architecture of distributed satellites for 5G/IoT. CEUR Workshop Proceedings. Proceedings of the International Workshop on Conflict Management in Global Information Networks (CMiGIN 2019) co-located with 1st International Conference on Cyber Hygiene and Conflict Management in Global Information Networks (CyberConf 2019). Lviv, 2588, 604-613. Available at: http://ceur-ws.org/Vol-2588/paper51.pdf 
[19] Buranova, M. A., Kartashevskyi, V. H., Samoilov, M. S. (2013). The comparative analysis of statistical characteristics of the video traffic in networks of the packet transmission of data. Infokommunikacionnye tehnologii, 11 (4), 33-39. Available at: https://readera.ru/read/140191662

[20] Sumtsov, D., Osiievskyi, S., Lebediev, V. (2018). Development of a method for the experimental estimation of multimedia data flow rate in a computer network. Eastern-European Journal of Enterprise Technologies, 2 (2 (92)), 56-64. doi: https:// doi.org/10.15587/1729-4061.2018.128045

[21] Ruban, I., Smelyakov, K., Martovytskyi, V., Pribylnov, D., Bolohova, N. (2018). Method of neural network recognition of ground-based air objects. 2018 IEEE 9th International Conference on Dependable Systems, Services and Technologies (DESSERT). doi: https://doi.org/10.1109/dessert.2018.8409200

[22] Mashtalir, S., Mikhnova, O., Stolbovyi, M. (2018). Sequence Matching for Content-Based Video Retrieval. 2018 IEEE Second International Conference on Data Stream Mining \& Processing (DSMP). doi: https://doi.org/10.1109/dsmp. 2018.8478597

[23] Piramanayagam, S., Saber, E., Cahill, N. D., Messinger, D. (2015). Shot boundary detection and label propagation for spatio-temporal video segmentation. Image Processing: Machine Vision Applications VIII. doi: https://doi.org/10.1117/ 12.2076661 